\title{
On the Feasibility of Mode-Division Multiplexed Transmission over Few-Mode Fibres
}

\author{
(Invited Paper)
}

\author{
Filipe M. Ferreira, Christian S. Costa, Stylianos Sygletos, Andrew D. Ellis \\ Aston Institute of Photonic Technologies, Aston University, Birmingham, B4 7ET, UK \\ f.ferreira@aston.ac.uk
}

\begin{abstract}
This paper reviews our most recent results on mode-division multiplexing systems based on few-mode fibres with low differential mode delay, spanning from the fibre design optimization to the receiver memory dimensioning. First, the fibre refractive-index profile is optimized for low differential mode delay and low macro-bend losses. Afterwards, we present a semi-analytical model of the linear mode coupling induced by fibre imperfections and stress. Finally, the interplay between the linear mode coupling and the differential mode delay is studied numerically and analytically, allowing to quantify the receiver memory required for a given fibre span.
\end{abstract}

Keywords-Few-Mode Fibres; Mode-Division Multiplexing; Differential Mode Delay; Linear Mode Coupling

\section{INTRODUCTION}

Mode-Division Multiplexing over few-mode fibres (FMFs) has been proposed as a next-generation solution to overcome the impeding exhaustion of the installed capacity using singlemode fibres (SMFs), with potential cost, space, and energy savings [1]. An $N$-fold capacity increase can be obtained using a few-mode fibre with $N$ independent modes, since each mode can support the same amount of information of a SMF. However, there are additional impairments arising from the multimode nature of FMFs that must be addressed to reach their full capacity [2]-[4]: differential mode delay (DMD), linear mode coupling (XT) and intermodal nonlinearities (IM-NL).

The combined effect of DMD and XT can be successfully compensated using multiple-input multiple-output (MIMO) equalization, but the additional processing complexity partially erodes the benefit of deploying FMFs [5]. One of the approaches proposed to reduce the equalizer complexity is the minimization of XT along the transmission system so that each mode can be detected individually without MIMO processing. However, the XT introduced by practical FMFs [6]-[9] would limit transmission distance to less than a couple of hundred kilometres. Other approach is to design low DMD fibres (but higher XT) requiring moderate MIMO processing complexity. For example, $D M D<12 \mathrm{ps} / \mathrm{km}$ over the C-band allows for MDM transmission over $2000 \mathrm{~km}$ at $100 \mathrm{~Gb} / \mathrm{s}$ [5]. Moreover, higher XT fibres have the added benefit of reducing the efficiency of IM-NL effects [10].

In this paper, we review our most recent results on MDM systems using low DMD FMFs. Section II reviews the design of fibres with a graded-index core for low DMD and with cladding trenches to reduce macro-bend losses (MBL) [11]. Section III reviews the semi-analytical model developed for
$\mathrm{XT}$ in FMFs [12]. Section IV reviews the impact of linear mode coupling on the statistics of mode group delays and on the receiver memory required for a given fibre span [13].

\section{LOW DMD FMF DESIGN}

We have selected a refractive-index profile with a gradedindex core and a cladding trench because the first has successfully been used to reduce DMD in conventional multimode fibres [14] and the later has been used to effectively reduce macro-bend losses [15].

The graded-core with cladding trench (GCCT) can be described using the refractive index relative difference $(\Delta n)$ function of the radial coordinate $\rho$ and given by $\Delta n(\rho)=\left[n(\rho)-n_{c l}\right] / n(\rho)$, where $n(\rho)$ is the refractive index at $\rho$ and $n_{c l}$ is the cladding refractive index. The guided modes $(\mathrm{LP} \mu v)$ and their characteristics are calculated solving the Maxwell equations numerically using the method described in [16]. The DMD of the LP $\mu \nu$ mode is measured relatively to the $\mathrm{LP}_{01}$ mode and the MBL is calculated according to [17]. The dispersion properties of the doped silica have been modelled using the Sellmeier coefficients provided in [18]. The GCCT profile is presented in Fig. 1 and analytically described by:

$$
n(\rho)=\left\{\begin{array}{ccc}
n(0)\left[1-\Delta n_{c o}\left(\rho / w_{1}\right)^{\alpha}\right], & |\rho|<w_{1} \\
n_{c l}, & w_{1} \leq|\rho|<w_{1}+w_{2} \\
n_{c l} /\left(1-\Delta n_{t r}\right), & w_{1}+w_{2} \leq|\rho|<w_{1}+w_{2}+w_{3} \\
n_{c l}, & |\rho| \geq w_{1}+w_{2}+w_{3}
\end{array}\right.
$$

where $w_{1}$ is the core radius, $w_{2}$ is the distance between the end of the core and the beginning of the trench, $w_{3}$ is the trench width, $\Delta n_{c o}=\Delta n(0)$, and $\Delta n_{t r}$ is $\Delta n$ at the trench. When designing a graded core fibre with $x$ modes $(x \mathrm{M})$, we choose the highest possible normalized frequency to guarantee the strongguidance of the first $x$-modes while cutting off the next modes.

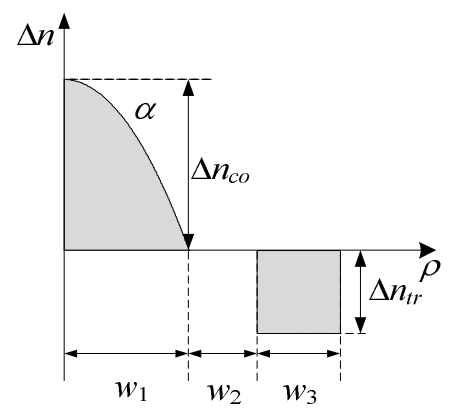

Fig. 1. Graded-index core with cladding trench refractive-index profile. 


\section{A. Profile Optimization and Optimization Algorithm}

Two of the most critical parameters in (1) are the defining shape power exponent, $\alpha$, and the trench depth, $\Delta n_{t r} . \alpha$ allows controlling the DMD average and $\Delta n_{t r}$ allows controlling the DMD slope [11]. Furthermore, the DMD can be decreased by simply reducing $\Delta n_{c o}$, since the modes effective-indexes are bounded by $n_{c o}$ and $n_{c l}$, thus the group-indexes defining the $D M D$ value. However, low $\Delta n_{c o}$ values lead to increased MBL. According to [17], the power loss at bends increases with decreasing $\Delta n_{c o}$ for a certain curvature radius. Thus, there is a trade-off between DMD and MBL on the optimization of $\Delta n_{c o}$.

The optimization function needs to consider two figures of merit: one related to DMD and another related to MBL. The DMD related figure is the maximum DMD among the guided modes over the defined wavelength range $(\max D M D)$, and the MBL related figure is the curvature radius $\left(R_{c}\right)$ for 100 turns and $M B L=0.1 \mathrm{~dB}$ at $1625 \mathrm{~nm}$. For a given $x \mathrm{M}$, the $R_{c}$ of each mode is calculated and the highest value is considered. Considering the ITU-T recommendation in [19], $R_{c}$ must be lower than or equal to $30 \mathrm{~mm}$. The optimization function $(O F)$ used in [11] is given by:

$$
\begin{aligned}
& \text { OF }(p v)=\max D M D(p v) \cdot\left\{1+\varepsilon \cdot\left[\beta \cdot \frac{\left(R_{c}-30\right)}{30}\right]\right\} \\
& p v=\left[\alpha, \Delta n_{c o}, \Delta n_{t r}, w_{2}, w_{3}\right] \\
& \beta=\left\{\begin{array}{l}
0, \text { for } R_{c} \leq 30 \\
1, \text { for } R_{c}>30
\end{array}\right. \\
& \text { with } R_{c} \text { in millimeter units. }
\end{aligned}
$$

where the $\varepsilon$ factor can be 0 or 1 to consider or ignore the $R_{c} \leq 30 \mathrm{~mm}$ requirement. The $\beta \cdot\left(R_{c}-30\right) / 30$ factor in (2) introduces a penalizing factor for solutions with $R_{c}>30 \mathrm{~mm}$, since $\beta$ is equal to 0 for $R_{c} \leq 30 \mathrm{~mm}$ and equal to 1 for $R_{c}>30 \mathrm{~mm}$. Note that, for every set of parameters $(p v)$ tested resulting in a number of modes different from the desired, the $O F$ value is set to infinity.

The optimization algorithm for the GCCT profile is designed to take advantage of the $\max D M D$ function properties. It can be shown that $\max D M D$ is a convex function of $\left(\alpha, \Delta n_{t r}\right)$ [11], therefore, the search for $\left(\alpha, \Delta n_{t r}\right)$ that minimizes $\max D M D$ for a given $\left(\Delta n_{c o}, w_{2}, w_{3}\right)$ point can be done one dimension at a time using, for example, a golden section search (GSS) [20]. Finally, to find the full optimum $p v$ set, an exhaustive search (ES) is performed over $\left(\Delta n_{c o}, w_{2}, w_{3}\right)$. The GSS optimizes $\alpha$ and $\Delta n_{t r}$ with a termination tolerance on $\max D M D$ of $0.001 \mathrm{ps} / \mathrm{km}$. The ES optimizes the $\Delta n_{c o}, w_{2}$ and $w_{3}$ with tolerances of $5 \cdot 10^{-4}, 0.25 \mu \mathrm{m}$ and $0.5 \mu \mathrm{m}$, respectively. Further reducing these tolerances by a factor of 2 changed $\max D M D$ negligibly.

\section{B. Optimization Results}

Fig. 2 (a) shows that $\max D M D$ decreases with decreasing $\Delta n_{c o}$ for all numbers of modes, in line with the explanation provided above, independently of $\varepsilon$. Fig. 2 (b) shows that $R_{c}$ increases when $\Delta n_{c o}$ decreases for $\varepsilon=0$, such that for $\Delta n_{c o}<5 \cdot 10^{-3}$ the $R_{c}$ requirement is not satisfied for all the numbers of modes considered. On the other hand, considering (a)

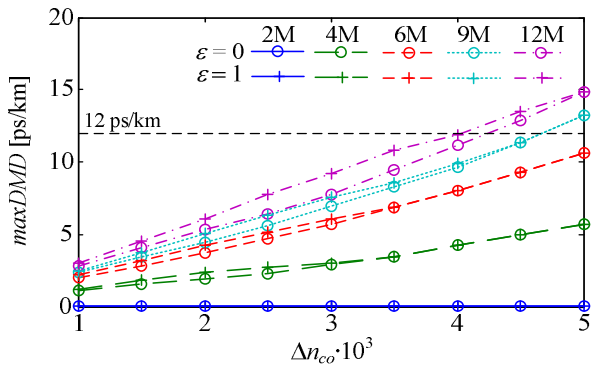

(b)

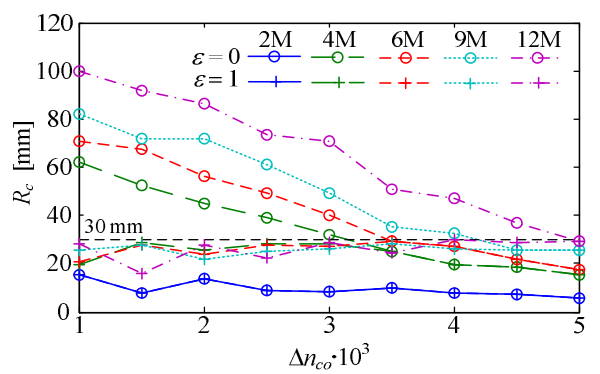

Fig. 2. (a) $\max D M D[\mathrm{ps} / \mathrm{km}]$ optimum values as a function of $\Delta n_{c o}$ for different $x \mathrm{M}$. (b) $R_{c}[\mathrm{~mm}]$ as a function of $\Delta n_{c o}$ for different $x \mathrm{M}$.

$\varepsilon=1$, Fig. 2 (b) shows that the $R_{c}$ requirement is satisfied for all $\Delta n_{c o}$ values and numbers of modes considered, $1 \cdot 10^{-3} \leq \Delta n_{c o} \leq 5 \cdot 10^{-3}$. Comparing the results shown in Fig. 2 (a) obtained using $\varepsilon=0$ and $\varepsilon=1$, it can be concluded that the $R_{c}$ requirement can be satisfied from $2 \mathrm{M}$ to $12 \mathrm{M}$ with small maxDMD degradation (lower than $0.5 \mathrm{ps} / \mathrm{km}$ for $\left.\Delta n_{c o}=1 \cdot 10^{-3}\right)$. Therefore, Fig. 2 shows that the $\max D M D$ and $R_{c}$ requirements are satisfied simultaneously for $1 \cdot 10^{-3} \leq \Delta n_{c o} \leq 4 \cdot 10^{-3}$ from $2 \mathrm{M}$ to $12 \mathrm{M}$. Moreover, it can be concluded that $\max D M D$ cannot be reduced to negligible levels $(\max D M D<0.1 \mathrm{ps} / \mathrm{km})$ for more than $2 \mathrm{M}$. This limitation is explained noting that the field confinement effect of the trench affects each higher-order mode (LP02, LP21, ...) with different strength, since all have a considerable power concentration near the core boundary but different distributions [21]. Therefore, each mode has a different optimum trench dimensioning and it is not possible to reduce the DMD of all modes to negligible values at the same time over the C-band. Moreover, increasing the number of modes, the $\left(\alpha, \Delta n_{t r}, w_{2}, w_{3}\right)$ domain satisfying $\max D M D \leq 12 \mathrm{ps} / \mathrm{km}$ decreases for a given $\Delta n_{c o}$.

The remaining fibre characteristic (besides $\max D M D$ and $R_{c}$ ) of the optimized fibres shown Fig. 2 are similar of that of conventional single-mode fibres. At $1550 \mathrm{~nm}$, the chromatic dispersion values are only slightly higher than $\sim 17 \mathrm{ps} /(\mathrm{nm} \cdot \mathrm{km})$, whereas the dispersion slope values are lower than $\sim 80 \mathrm{fs} /\left(\mathrm{nm}^{2} \cdot \mathrm{km}\right)$. The $\gamma$ value is significantly lower than the SSMF typical value of $1.3 \mathrm{~W}^{-1} / \mathrm{km}$, as expected due to the higher core radius.

The results presented in this section allow stating that optimizing $\Delta n_{c o}$ allowed to fulfil the requirement of $\max D M D \leq 12 \mathrm{ps} / \mathrm{km}$ and $R_{c} \leq 30 \mathrm{~mm}$, which was not achievable in [21]. However, the optimized profiles presented above were obtained ignoring the finite precision margins of the manufacturing control processes. These margins lead to deviation of the manufactured fibre parameters from the 
(a)

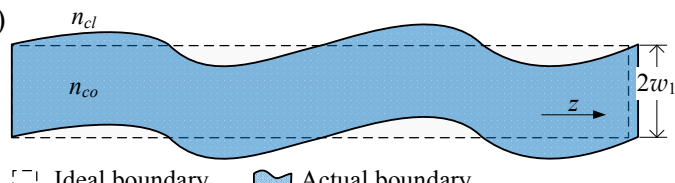

[-- Ideal boundary $\cong$ Actual boundary

(b)

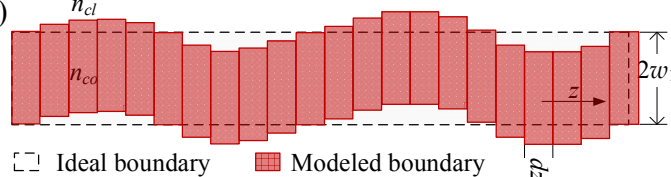

Fig. 3. Fiber dielectric waveguide with distorted core-cladding boundary.

optimum values, causing DMD deviations. Considering that parameters deviations on conventional multimode fibres are well described by normal distributions with known standard deviation [22], the manufacturing success probability can be assessed. It can be shown that for $\Delta n_{c o}=2 \cdot 10^{-3}$, success that probability is higher than $95 \%$ for $2 \mathrm{M}$. But closer to $68 \%$ for $4 \mathrm{M}$ to $12 \mathrm{M}$ cases [11].

\section{COUPLED-Mode THeORY FOR FeW-Mode Fibres}

In this section, we review the linear mode coupling model [12]. The model describes the fibre imperfections responsible for mode coupling as random fluctuations of the core centre position, given by: $\varepsilon(x, y, z)=\varepsilon_{p}(x+\delta x(\mathrm{z}), y+\delta y(\mathrm{z}), z)$, where $\varepsilon$ is the perturbed permittivity, $\varepsilon_{p}$ is the ideal relative permittivity, $\delta x$ and $\delta y$ are the random displacement of the abscissa and ordinate coordinates, respectively. The proposed model divides the fibre in multiple sections of length $L_{s}$, each with a constant random displacement of the core centre position, as shown in Fig. 3. Therefore, each section has constant coupling coefficients. To analytically describe the coupling arising from the waveguide imperfections, we used the coupled wave theory [2], particularly the following coupled-mode equations:

$$
\begin{aligned}
& \partial_{z} \tilde{A}_{\mu}(z, \omega)=-\mathrm{j}\left[\beta_{\mu}(\omega)-\beta_{0 \mu}\right] \tilde{A}_{\mu}(z, \omega) \\
&-\mathrm{j} \sum_{v \neq \mu} C_{\mu \nu}(z) \tilde{A}_{v}(z, \omega) \exp \left[\mathrm{j}\left(\beta_{0 \mu}-\beta_{0 v}\right) z\right] \\
& C_{\mu \nu}(z)=\frac{\omega \varepsilon_{0}}{4} \iint_{-\infty}^{+\infty}\left[\varepsilon(x, y, z)-\varepsilon_{p}(x, y, z)\right] \mathbf{E}_{\mu} \cdot \mathbf{E}_{v}^{*} d x d y
\end{aligned}
$$

where $\tilde{A} \mu(z, \omega)$ is the Fourier transform of the mode $\mu$ slowly varying field envelope $A_{\mu}, \beta_{\mu}(\omega)$ is the mode $\mu$ propagation constant, and $\beta_{l \mu}$ is the $l^{\text {th }}$ order coefficient of a Taylor series expansion of $\beta_{\mu}(\omega)$ centred at the carrier frequency $\omega_{0}$. $C_{\mu v}$ are the coupling coefficients given by the area integral of the dot product of the electrical fields of mode $\mu$ and mode $v$, over the area where the permittivity difference is nonzero.

In FMFs, the mode coupling strength can be quantified as the ratio between the average power in all the other orthogonal modes and average power remaining in the launching mode, after a certain distance, this is:

$$
X T_{m}=\sum_{v \neq \mathrm{m}}\left(P_{v} / P_{m}\right)
$$

where $P_{v}$ is the power of mode $v$, after a given fibre segment under test, when only $m$ mode was launched. Thus, there are as many coupling strength values and $L_{c}$ as the number of modes.

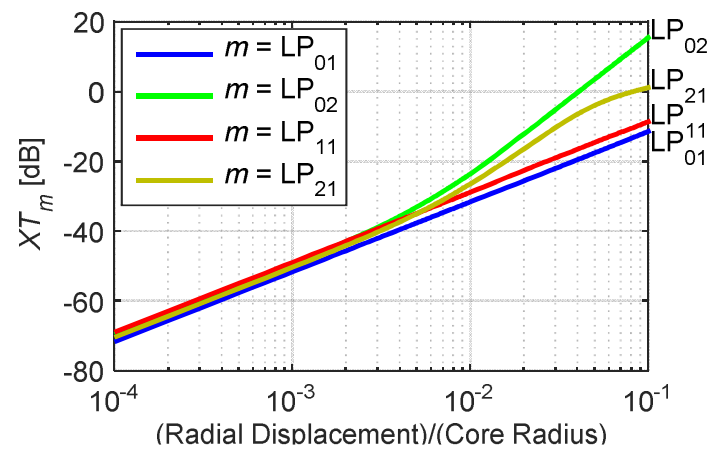

Fig. 4. $X T_{m}$ averaged over the azimuth displacement as a function of the radial displacement

Inevitable, the mode $m$ showing higher coupling strength will set the reference for the study of the group-delay statistics.

The statistical nature of GDs in FMFs is mainly determined by $X T_{m}$ or the equivalent correlation length $\left(L_{c}\right)$, similarly to SMFs [24]. In FMFs, the correlation length is defined as the fibre length for which $\left(P m-\sum_{v \neq \mathrm{m}} P_{v}\right)=e^{-2}$, equivalent to $X T_{m}=\left[e^{2}-1\right] /\left[e^{2}+1\right](-1.18 \mathrm{~dB})$. The coupling regimes may then be defined as strong coupling for $L \gg L_{c}$, weak coupling for $L<<L_{c}$, and intermediate coupling otherwise. In the literature, the mode coupling values of fabricated FMFs range from $-50 \mathrm{~dB} / 100 \mathrm{~m}$ to $-40 \mathrm{~dB} / 100 \mathrm{~m}$ for fibres with step-index or graded-index profiles [6]-[7], going up to $-28 \mathrm{~dB} / 100 \mathrm{~m}$ for coupled multi-core fibres [8] and $-7 \mathrm{~dB} / 100 \mathrm{~m}$ for fibres with ring-index profiles [9]. For $X T$ values range from -40 to $-30 \mathrm{~dB} / \mathrm{km}$, and the respective $L_{c}$ values (given by $L_{c}$ definition) range from 7,225 to $800 \mathrm{~km}$. Consequently, for typical fibre link distances, less than $1000 \mathrm{~km}$ [23], the system is neither operating in the strong coupling regime nor the weak coupling regime $\left(L \sim L_{c}\right)$. Thus, the performance of FMF systems, for these mode coupling levels and typical distances, might not be accurately modelled by random unitary matrices (every $\sim 100 \mathrm{~m}$ ) because the impact of group delay spread and intermodal nonlinearity on the performance might be underestimated [10].

Finally, the linear transmission matrix for a FMF can be obtained by numerically solving (3) or by using the semianalytical solutions presented in [12]. Fig. 4 shows the $X T$ as function of the core displacement for a fibre segment with one single displacement, considering the 4M-FMF optimized for $\Delta n_{c o}=4.5 \cdot 10^{-3}$ in Section II. In Fig. 4 , the mode coupling strength only depends significantly on $m$ for displacements higher than $1 \%$. Such higher coupling for $\mathrm{LP}_{02}$ and $\mathrm{LP}_{21}$ can be explained noting they belong to the same LP mode group. Moreover, $X T_{\mathrm{LP} 21}<X T_{\mathrm{LP} 02}$ for any displacement in Fig. 4 because any power launched in $\mathrm{LP}_{21 \text { a }}$ couples preferentially with $\mathrm{LP}_{21 \mathrm{~b}}$ (and vice-versa) and in the second place to $\mathrm{LP}_{02}$. Given the higher values of $X T_{\mathrm{LP} 02}$, we define $L_{c}$ for this mode. Note that $X T_{m}$ values above $10 \mathrm{~dB}$ mean that almost all power launched in mode $m$ has been transferred to other modes.

\section{Mode COUPLING IMPACT ON GROUP-DELAYS}

The statistical properties of the GDs are well known for the two extreme regimes [25]-[26]. In the weak coupling regime 


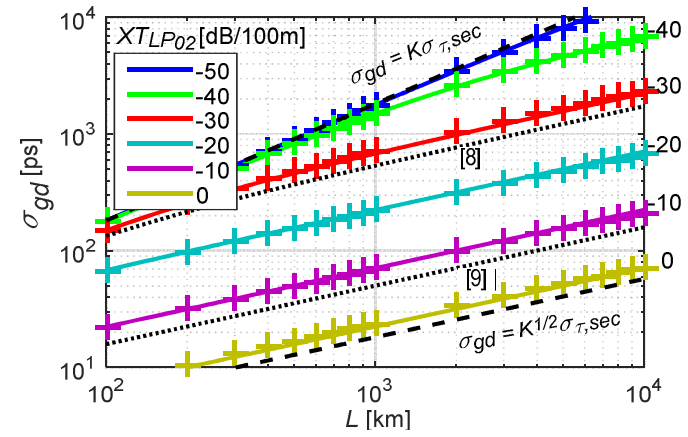

Fig. 5. $\sigma_{g d}$ as a function of transmission distance showing simulation results (markers) and analytical results (solid lines).

$\left(L<<L_{c}\right)$, the GD spread grows linearly with distance and in the strong coupling regime $\left(L>>L_{c}\right)$ grows with the square root of the distance. In the intermediate coupling regime, we shown in [13] through simulation that the GDs statistics in SMFs can be extended to FMFs, at least for fibres guiding 6 LP modes. At the same time, the complete analytical derivation of such extension was presented in [24]. In the intermediate coupling regime, the standard deviation of the GDs in FMFs can be written as [13], [24]:

$$
\sigma_{g d}=\sqrt{2} \sigma_{\tau} L_{c}\left(e^{-L / L_{c}}+L / L_{c}-1\right)^{1 / 2}
$$

where $\sigma_{\tau}$ is the standard deviation of the uncoupled GDs per unit length.

In the following, the impact of linear mode coupling on the GDs spread is evaluated. The 4M-FMF optimized for $\Delta n_{c o}=4.5 \cdot 10^{-3}$ in Section II is considered here, it guides six modes (LP01, LP02, LP11a, LP11b, LP21a, LP21b) and presents a DMD of $5.19 \mathrm{ps} / \mathrm{km}$. The $X T_{\mathrm{LP} 02}$ value was varied from -50 to $0 \mathrm{~dB} / 100 \mathrm{~m}$ by varying the amplitude of the variation in lateral section offset, as explained in Section III, assuming a section length of $100 \mathrm{~m}$. This range fully covers the range of coupling values presented in the literature [6]-[9]. Finally, the GDs were calculated through direct numerical solution of the coupled-mode equations describing the linear mode propagation [12].

Fig. 5 shows the standard deviation of the GDs of the PMs $\left(\sigma_{g d}\right)$ as a function of distance up to $10,000 \mathrm{~km}$, obtained by averaging over 2000 different realizations of lateral offsets giving rise to a given $X T_{\mathrm{LP}_{02}}$ value. Fig. 5 shows a good agreement between numerical simulation and the analytical expression (6), for any coupling value studied and for any distance up to $10,000 \mathrm{~km}$. In Fig. 5, for coupling values ranging from -50 to $-40 \mathrm{~dB} / 100 \mathrm{~m}, \sigma_{g d}$ scales approximately linearly with distance. But, at $-40 \mathrm{~dB} / 100 \mathrm{~m}$ the deviation from linear growth is already noticeable above $1,000 \mathrm{~km}$, thus even with such a low coupling, the FMF is operating in intermediate coupling regime. Increasing $X T_{\mathrm{LP}_{02}}, \sigma_{g d}$ gradually converges to the strong coupling regime. However, even for a $X T_{\mathrm{LP}_{02}}$ equal to $-7.01 \mathrm{~dB} / 100 \mathrm{~m}$ (the highest value found in literature [9]) the fibre is still not fully operating in the strong coupling regime when considering a section length of $100 \mathrm{~m}$. In this case, assuming strong coupling regime (random unitary matrices every $100 \mathrm{~m}$ ), would underestimated $\sigma_{g d}$ by a factor of 2.76 .
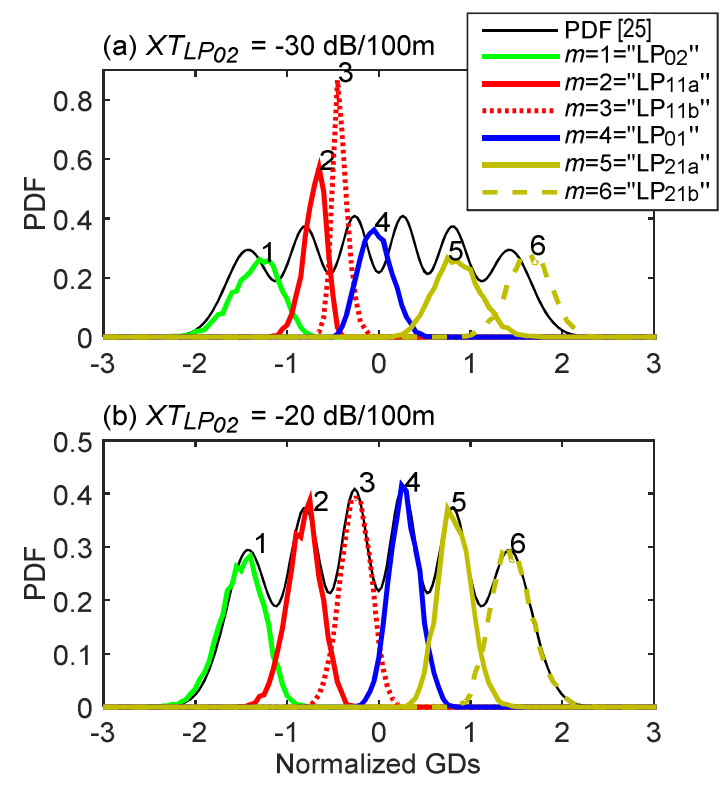

Fig. 6. PDF of the ordered normalized GDs $\left(\tau_{m} / \sigma_{g} d\right)$, obtained through simulation after $1000 \mathrm{~km}$, with different $X T_{\mathrm{LP} 02}$ values.

Fig. 6 shows the PDF of the ordered GDs $\left(\tau_{m}\right.$, $\left.\tau_{1} \leq \tau_{2} \leq \ldots \leq \tau_{6}\right)$, normalized by $\sigma_{g d}$, after $1000 \mathrm{~km}$ for two different coupling values, overlapped with the analytical joint PDF (thin black line) derived for the strong coupling regime [25]. Note that the normalization factor $\left(\sigma_{g d}\right)$ depends on the $X T_{\mathrm{LP}_{02}}\left(L_{c}\right)$ value, see (6). Fig. 6 (a) shows that for $-30 \mathrm{~dB} / 100 \mathrm{~m}$ the GDs of the PMs vaguely resemble the GDs of the LP modes given the impulse-like PDF of $\tau_{2}$ ("LP ${ }_{11 \mathrm{a}}$ ") and $\tau_{3}$ ("LP ${ }_{11 \mathrm{~b}}$ "). Further results for lower coupling values shown that all GDs present impulse-like PDFs. In Fig. 6(b), for $-20 \mathrm{~dB} / 100 \mathrm{~m}$, the match between the simulated PDFs and the analytical PDF for strong coupling is good, even though the GDs have been normalized by different factors given by (6). Further results shown that the match between the simulated PDFs and the analytical PDF improves for higher coupling values.

In a MDM system, to fully compensate for DMD and mode coupling, the MIMO equalizer must span a temporal memory at least as long as the system GD spread $\left(\tau_{6}-\tau_{1}\right)$. Fig. 7 shows the complementary cumulative distribution function (CCDF) of the normalized GD spread, $P\left[\left(\tau_{6}-\tau_{1}\right) / \sigma_{g d}>p\right]$, obtained through simulation after $1000 \mathrm{~km}$ for different coupling values (averaging over 6000 different realizations). Fig. 7 shows that for $X T_{\mathrm{LP}_{02}} \geq-30 \mathrm{~dB} / 100 \mathrm{~m}$ the CCDFs are very similar to the analytical approximation obtained for strong coupling [28] (dashed line). Conversely, for $X T_{\mathrm{LP} 02}$ lower than $-30 \mathrm{~dB} / 100 \mathrm{~m}$ the normalized GD spread is significantly smaller than the normalized GD spread for strong coupling.

Combining this observation with the normalisation factor, given by (6), we find that the required temporal equalizer memory length $\left(\Delta T_{E Q}\right)$ to span the channel memory with a given probability $p$ and for a given mode coupling strength is given by (in time units): 


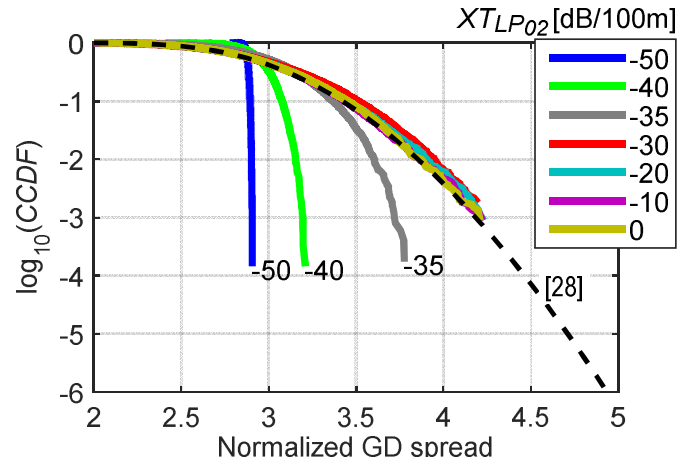

Fig. 7. Complementary cumulative distribution of the normalized GD spread, obtained through simulation after $1000 \mathrm{~km}$, while varying $X T_{\mathrm{LP} 02}$.

$$
\Delta T_{E Q} \cong U(p) \cdot \sigma_{g d}=U(p) \cdot \sqrt{2} \sigma_{\tau} L_{c}\left(e^{-L / L_{c}}+L / L_{c}-1\right)^{1 / 2}
$$

where $U(p)$ can be calculated using eq. 6 in [28], dashed line in Fig. 7. A $\Delta T_{E Q}$ from $4 \sigma_{g d}$ to $5 \sigma_{g d}$ is sufficient to span the channel memory with probability from $10^{-4}$ to $10^{-6}$.

\section{CONCLUSIONS}

Along this paper, we reviewed our most recent results on MDM systems based on low DMD FMFs. First, we demonstrated that FMFs guiding up to 12 non-degenerate LP modes can be designed with $D M D<12 \mathrm{ps} / \mathrm{km}$ and curvature radius of $30 \mathrm{~mm}$ by optimizing a graded-index with cladding trench profile. Using this profile, MDM transmission over $2000 \mathrm{~km}$ at $100 \mathrm{~Gb} / \mathrm{s}$ is feasible for current DSP complexity capabilities [5]. Afterwards, we presented a semi-analytical model for the linear mode coupling induced by fibre imperfections and stress, a valuable alternative to the numerical methods impractical for long-haul simulation due to computation time constraints. The proposed model proved accurate against analytical predictions for the statistics of GDs in FMFs, namely: standard deviation, probability density function, and cumulative distribution function. Finally, the proposed semi-analytical solution method is an accurate and efficient tool for the modelling and development of future highcapacity multimode fibre systems.

\section{ACKNOWLEDGMENT}

This work has been partially supported by European Union (Grants 619732-INSPACE, 654809-HSPACE and 659950INVENTION), and by EPSRC (Grant EP/L000091/1-PEACE).

The Matlab scripts, source C-code, mex compiled C-code, and figure data points are available at http://researchdata.aston.ac.uk.

\section{REFERENCES}

[1] A. D. Ellis, J. Zhao and D. Cotter, "Approaching the Non-Linear Shannon Limit," Journal of Lightwave Technology, vol. 28, no. 4, pp. 423-433, 2010.

[2] D. Marcuse, Theory of Dielectric Optical Waveguides, New York: Academic, 1974, Chap. 3 and 5

[3] K.-P. Ho and J. M. Kahn, "Statistics of Group Delays in Multimode Fiber with Strong Mode Coupling", Journal of Lightwave Technology, vol. 29 , no. 21 , pp. 3119-3128, 2011.
[4] S. Mumtaz, R. J. Essiambre and G. P. Agrawal, "Nonlinear Propagation in Multimode and Multicore Fibers: Generalization of the Manakov Equations," Journal of Lightwave Technology, vol. 31, no. 3, pp. 398406, Feb.1, 2013.

[5] B. Inan, B. Spinnler, F. Ferreira, et al., "DSP complexity of modedivision multiplexed receivers," Opt. Express, vol. 20, p. 10859, 2012.

[6] L. Grüner-Nielsen, et al., "Few Mode Transmission Fiber With Low DGD, Low Mode Coupling, and Low Loss," J. Lightw. Technol., Vol. 30, no. 23, p. 3693, 2012.

[7] T. Mori, et al., "Low DMD Four LP Mode Transmission Fiber for Wideband WDM-MIMO System,” Proc. OFC 2013, p. OTh3K.1.

[8] R. Ryf, R.-J. Essiambre, A. H. Gnauck, S. Randel, et al., "Space-division multiplexed transmission over 4200-km 3-core microstructured fiber," in Proc. OFC 2012, p. PDP5C.2.

[9] N. Fontaine, et al., "Experimental investigation of crosstalk accumulation in a ring-core fiber," in Proc. PSSTMS 2013, p. TuC4.2.

[10] F. Ferreira, N. Mac Suibhne, C. Sánchez, S. Sygletos, and A. D. Ellis, "Advantages of Strong Mode Coupling for Suppression of Nonlinear Distortion in Few-Mode Fibers," OSA2016, paper Tu2E.3

[11] F. Ferreira, D. Fonseca and H. Silva, "Design of Few-Mode Fibers With M-modes and Low Differential Mode Delay," Journal of Lightwave Technology, vol. 32, no. 3, pp. 353-360, 2014.

[12] F. Ferreira, S. Jansen, P. Monteiro and H. Silva, "Nonlinear SemiAnalytical Model for Simulation of Few-Mode Fiber Transmission," in IEEE Photonics Technology Letters, vol. 24, no. 4, pp. 240-242, 2012.

[13] F. Ferreira, N. Suibhne, S. Sygletos and A. D. Ellis, "Few-mode fibre group-delays with intermediate coupling," ECOC 2015, p. Th.1.6.1

[14] P. Matthijsse, D. Molin, F. Gooijer, and G. Kuyt, "On the design of wide bandwidth window multimode fibers," IWCS 2005, pp. 332-337.

[15] P. Watekar, S. Ju, Y. Yoon, Y. Lee, and W. Han, "Design of a trenched bend insensitive single mode optical fiber using spot size definitions," Opt. Express 16, 13545-13551 (2008)

[16] C. Yeh, and G. Lindgren, "Computing the propagation characteristics of radially stratified fibers: an efficient method," Appl. Opt., vol. 16, no. 2, pp. 483-493, 1977.

[17] J. Sakai, and T. Kimura, "Bending loss of propagation modes in arbitrary-index profile optical fibers," Appl. Opt., vol. 17, no. 10, pp. 1499-1506, 1978

[18] W. Hermann, and D. U. Wiechert, "Refractive index of doped and undoped PCVD bulk silica," Mater. Res. Bull., vol. 24, no. 9, pp. 1083 1097, 1989

[19] Characteristics of a single-mode optical fibre cable, Standard ITU-T G.652B, Oct. 2010.

[20] W. Press, S. Teukolsky, W. Vetterling, and B. Flannery, Numerical Recipes: The Art of Scientific Computing, New York: Cambridge University Press, third edition, 2007.

[21] F. Ferreira, D. Fonseca, and H. Silva, "On the Dependence of Differential Mode Delay in Few-Mode Fibers on the Number of Modes," in Proc. ICTON 2013, pp. 1-4, paper Tu.C2.3.

[22] Krawarik P., Watkins L., "Fiber geometry specifications and its relations to measured fiber statistics", Applied Optics, 17(24), 3984-3989. 1978.

[23] S. Routray, et al., J. Opt. Commun. Netw., 5(7), p. 762, 2013.

[24] S. Fan and J. Kahn, "Principal modes in multimode waveguides," Opt. Lett., vol. 30, no. 2, p. 135, 2005.

[25] K.-P. Ho and J. M. Kahn, "Statistics of Group Delays in Multimode Fiber with Strong Mode Coupling", Journal of Lightwave Technology, vol. 29, no. 21, pp. 3119-3128, 2011.

[26] A. Mecozzi, C. Antonelli, and M. Shtaif, "Intensity impulse response of SDM links," Opt. Express vol. 23, pp. 5738-5743, 2015.

[27] S. Ö. Arik, K.-P. Ho and J. M. Kahn, "Delay Spread Reduction in ModeDivision Multiplexing: Mode Coupling versus Delay Compensation", J. of Lightwave Technol., vol. 33, no. 21, pp. 4504-4512, 2015.

[28] K. Ho, et al., "Delay-Spread Distribution for Multimode Fiber With Strong Mode Coupling," Photon. Technol. Lett., Vol. 24, no. 21, p. 1906, (2012). 